\title{
Optimization of Bus Route Planning in Urban Commuter Networks
}

\author{
Steven I-Jy Chien, Branislav V. Dimitrijevic, and Lazar N. Spasovic \\ New Jersey Institute of Technology
}

\begin{abstract}
Bus routing is one of the most important elements of public transit system planning. This article presents a model for optimizing service headway and a bus route serving an area with a commuter (many-to-one) travel pattern. The bus route is optimized by minimizing the total system cost, including operator and user costs, while considering diagonal links in the study network. A method is developed for transforming this network into a pure grid, which enables construction of pure grid network models applicable to irregular grid networks. A case is presented to demonstrate the application of the model. Results show that the optimal bus route is sensitive to demand distribution over the service area. The developed model is particularly useful for planning a new bus service and evaluating an existing one in many cities embedded with general grid networks.
\end{abstract}

\section{Introduction}

Commuter bus routes are generally located on main thoroughfares of urban areas. However, considering realistic distributions of passenger travel demand over space and time, many route locations may not be cost-effective from either the operator or user standpoint. Therefore, relocating bus routes and redesigning headways may reduce operating costs as well as improve passenger accessibility.

Both transit operators and passengers prefer short and fast routes to reduce the operating cost and travel time, respectively. However, passengers also prefer bus routes that can be easily accessed from their origins and destinations. To reduce 
access impedance, tortuous routes are often constructed. This, in turn, is likely to increase both the in-vehicle portion of user travel time as well as the bus operating cost. Transit operators are well aware of this trade-off when planning a new bus route or extending an existing service.

In the past 30 years, many researchers have analyzed the problems of optimal transit service design with many-to-one travel patterns by using analytical methods (Byrne and Vuchic 1971; Chang and Schonfeld 1991; Hurdle 1973; Spasovic and Schonfeld 1993; Spasovic et al.1994; Wirasinghe et al. 1977). They dealt with selecting zones, route/line spacings, headways, and route lengths designed to carry people between distributed origins and a single destination (e.g., central business district [CBD], transfer station, etc.). By assuming demand homogeneity of the service area, the researchers optimized the characteristics of bus systems consisting of a set of parallel routes feeding a major transfer station of a trunk line or a single terminal point, such as the CBD.

A recent method for analyzing fixed-route bus systems is the out-of-direction (OOD) technique (Welch et al. 1991). This method improves the accessibility of a bus system by improving passenger accessibility along certain route segments. Chien and Schonfeld (1997) optimize a grid transit system in an urban area without oversimplifying the spatial and demand characteristics. They extended the model to jointly optimize the characteristics of a rail transit route and the associated feeder bus routes in an urban corridor (Chien and Schonfeld 1998).

Chien and Yang (2000) developed an algorithm to search for the best bus route feeding a major intermodal station while considering the intersection delays and realistic street network. The model optimized the bus route location and operating headway by minimizing the sum of operator and user costs. It considered irregular and discrete demand realistically distributed over the service area. The route and headway were optimized analytically.

In marked contrast to the above research, this article deals with the irregular grid street network, including diagonal streets and heterogeneous demand over the service area. To formulate bus routing problems, diagonal streets are transformed into horizontal and vertical links so the grid structure of the network could be preserved to facilitate the computational process. Actual lengths of diagonal links are taken into account when calculating the route length and travel times. The bus route, headway, and fleet size are optimized by minimizing the total cost (the sum 
of operator and user costs). A computer program is developed to search for the optimal solution.

\section{Assumptions}

The commuter network discussed in this article is a general grid network with some diagonal links. To formulate the mathematical optimization model for such a network, the following assumptions are made:

- The irregularly shaped service area can be divided into many zones according to the street spacing and demand distribution.

- A feeder bus route provides service between the suburban area and the $\mathrm{CBD}$ (Figure 1a). Thus, the travel demand pattern of the area is many-toone in the morning peak period and one-to-many during the afternoon peak period.

- A line-haul distance J, connecting the CBD (or a major transfer station) and the service area at an entry point, is assumed to be constant.

- The demand is not sensitive to bus service quality or fare and is uniformly distributed within each zone. The zones may have different associated cost, demand, land-use, and traffic characteristics.

- Buses can stop anywhere along the route whenever a boarding or an alighting is requested by a passenger. Thus, the bus stop location can be ignored.

- Passengers access the route randomly, while the headway is short enough to assume that average wait time is half of the headway.

- The value of time is assumed to be additive. This assumption can be relaxed as long as the function of time value can be developed.

- The intersection (or node) delay incurred by bus is constant regardless of the bus size but may vary at different intersections.

- Vehicle layover time is negligible. 
Figure 1a: Graphical Interpretation of the Street Network

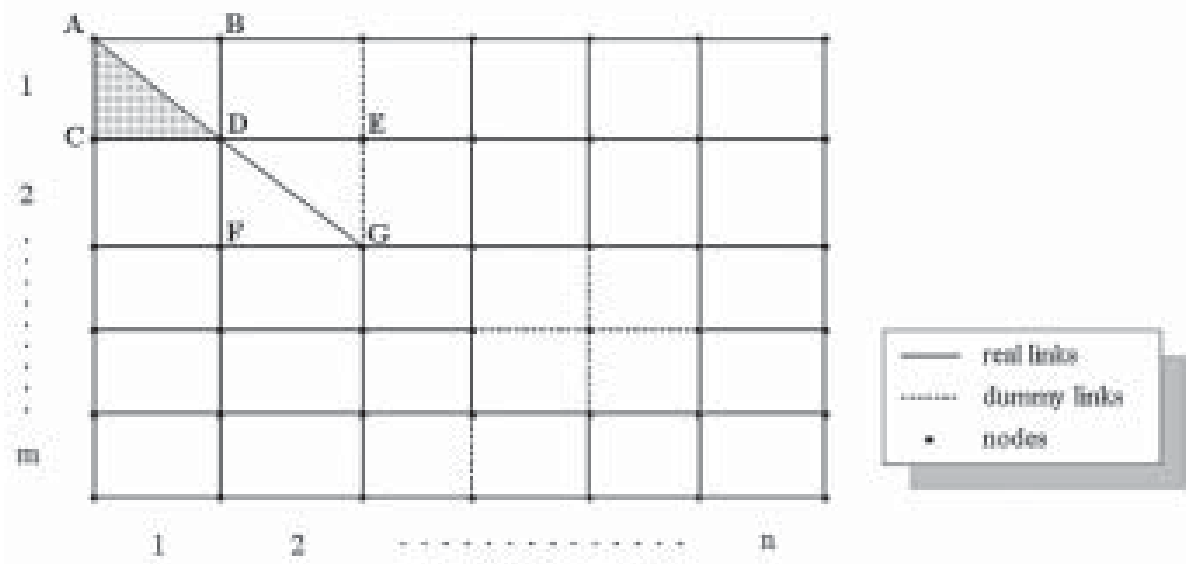

There are two types of links classified in the study network: real links and dummy links. Real links represent actual streets with the network. Dummy links do not exist in reality but are included to normalize the grid network structure. The length of dummy links is assumed to be infinite. Thus, in a minimization problem it will generate an infinite penalty for vehicles traveling through these links.

A diagonal link makes "triangular areas"_ with adjacent streets as shown in Figure 1a (shaded triangle A-C-D). The diagonal links are converted into horizontal and vertical links, as shown in Figure 1b. Diagonal links (AD and DG) have been replaced by horizontal links ( $X Y$ and $M N$ ), while the link length remains the same. When calculating the total route length, the distance between the previous node (e.g., A) and the incident node of the horizontal link (e.g., $X)$ is equal to zero $(A X=0)$. The same situation holds for link $Y D(Y D=0)$. However, the route that includes link $X Y$ must also contain links $A X$ and $Y D$. The length of link $X C$ is assumed to be equal to the actual length of the vertical link originally connecting nodes $A$ and $C$. The same situation holds for link BY (which has the length of the vertical link originally connecting nodes $B$ and $D)$.

As part of the transformation, new links marked as "dummy links" are introduced as extensions of links $X Y$ and $M N$ (e.g., L1, L2, L3, . ., L10, etc.) to preserve the grid network structure. Since new nodes are introduced in this transformation, intersection delay times for these nodes must also be defined. The intersection delay for node $X$ is equal to the intersection delay of node $A$. In the same manner, the 


\section{Figure 1b: Transformed Network Representation}

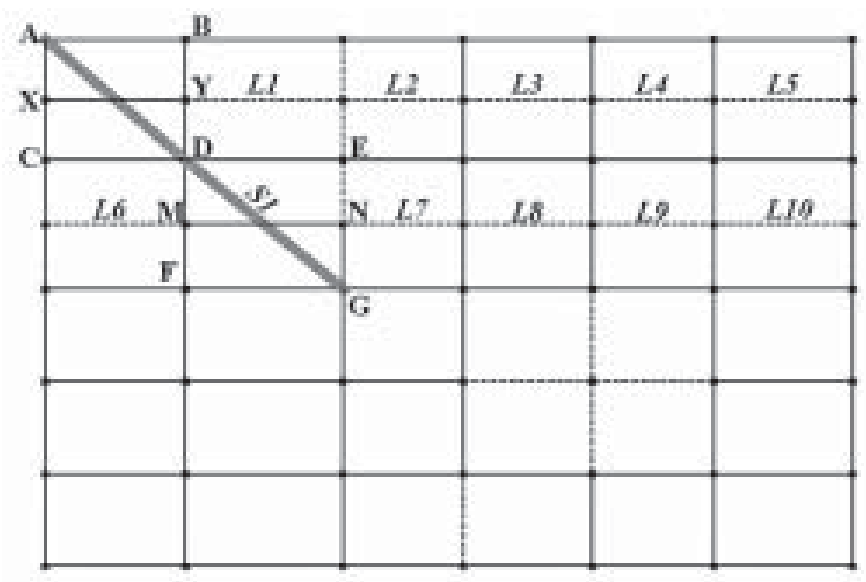

intersection delays of nodes $Y$ and $M$ are equal to the intersection delay of node $D$, while the intersection delay of node $N$ is equal to that of node $G$. To avoid double counting the intersection delay (e.g., $\mathrm{Y}, \mathrm{D}$, and $\mathrm{M}$ ), only intersection delay at the immediate downstream node of a link is considered when the route travel time is calculated. Delays at intersections of dummy links are set to be zero.

\section{Model Formulation}

The mathematical notation and the definition of variables and parameters used in this section are summarized in Table 1. The network, shown in Figure 1a, is divided into $m$ rows and $n$ columns, containing $m \times n$ zones (Figure 1b). The location of each zone is defined by indices of rows and columns. Demand of a zone, denoted by $q_{i j}(1 \leq i \leq m, 1 \leq j \leq n)$, is defined by the number of passengers of a particular zone. 
Journal of Public Transportation,Vol. 6, N 0. 1, 2003

\section{Table 1. Variable and Parameter Definition}

\section{Symbol Definition Unit}

$a_{w} \quad$ Total access time for passengers originating from zono (i.j) hours

$A_{0}^{X} \quad$ Network horizontal links matrix

$A_{j}^{Y} \quad$ Network vertical links matrix

$B_{L} \quad$ Network node matrix

$C_{A} \quad$ Total passenger access cost S/hour

$C_{s} \quad$ Total supplier cost S/hour

$\begin{array}{lll}C_{T} & \text { Total system cost } & \text { S/hour }\end{array}$

$C_{U} \quad$ Total user cost S/hour

C Vehicle capacity pass/bus

$C_{y} \quad$ Total in-vehicle cost S/hour

$C_{\mathrm{W}} \quad$ Total wait cost $\quad$ S/hour

$D_{i j} \quad$ Minimum access distance for passengers from zone $(i f) \quad \mathrm{km}$

$F$ Fleetsize buses

$8 \quad$ Passenger walking speed $\mathrm{km} / \mathrm{hour}$

$H_{N}$ Headway hours

$L_{j} \quad$ Line haul distance $\mathrm{km}$

d Length of the horizontal link that replaced the diagonal link km

$\ell \quad$ Length of the vertical link modified after diagonal link lom is replaced

$m \quad$ Number of rows in the network

$n \quad$ Number of columns in the network

$P_{v}^{x} \quad$ Penalty matrix for horizoneal links 
$P_{y}^{Y} \quad$ Penalty matrix for vervical links

$Q \quad$ Total passenger demand

pass/hour

$q_{i j} \quad$ Passenger demand of the zone $(i, j)$

pass/hour

$S_{D} \quad$ Total intersection delay incurred by passengers

hours

$S$, Total line haul in-vehicle time

hours

$S_{\ell} \quad$ Total route link in-vehicle time

hours

$S_{y} \quad$ Total in-vehicle time

hours

$T_{D} \quad$ Total route intersection delay

hours

$T_{\bar{y}} \quad$ Intersection delay matrix

hours

T) Line haul travel time

hours

$T_{L} \quad$ Total route link travel time

hours

$T_{\mathcal{R}} \quad$ Round trip travel time

hours

$u_{A}$

Value of passenger access time

\$/hour

$u_{B}$

Average bus operating cost

\$ihour

$u_{l} \quad$ Value of passenger in-vehicle time

\$/hour

$u_{w} \quad$ Value of passenger wait time

Shour

$V_{g} \quad$ Average bus operating speed in the service area

km/hour

$V_{J} \quad$ Line haul speed

km/hour

$X_{y} \quad$ Matrix of lengths of horizontal links in the network

$\mathrm{km}$

$X_{j}^{N}$

Width of the row $j$ of the network

$\mathrm{km}$

$Y_{i j} \quad$ Matrix of lenguts of wertical links in the network

$\mathrm{km}$

$Y_{y}^{N} \quad$ Width of the column i of the network

$\mathrm{km}$ 
Journal of Public Transportation,Vol. 6, N o. 1, 2003

The following matrices define horizontal and vertical links, and network nodes.

\section{Horizontal Links}

(a)

$$
\begin{aligned}
& A_{j j}^{X} \text { for } 1 \leq i \leq(m+1) \text { and } 1 \leq j \leq n \text { represents matrix of horizontal } \\
& \text { links } \\
& A_{i j}^{X}= \begin{cases}1, & \text { if the bus route; } \\
0, & \text { otherwise. }\end{cases}
\end{aligned}
$$

(b) $\quad X_{i j}$ for $1 \leq i \leq(m+1)$ and $1 \leq j \leq n$ represents matrix of horizontal link lengths

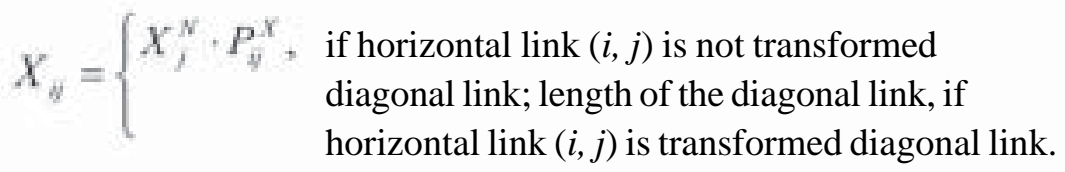

where:

$$
\begin{aligned}
& P_{\dot{y}}^{X} \quad \begin{array}{l}
\text { takes the value of } 1 \text { or }+\infty \text { depending on whether the } \\
\text { horizontal link }(i+j) \text { is a real or dummy link. }
\end{array} \\
& X_{j}^{N} \text { represents the width of the column } j .
\end{aligned}
$$

$$
\begin{gathered}
P_{\psi j}^{X} \begin{array}{l}
\text { for } 1 \leq i \leq(m+1) \text { and } 1 \leq j \leq n \text { represents penalty } \\
\text { matrix for horizontal links }
\end{array} \\
P_{j}^{x}= \begin{cases}+\infty, & \text { dummy link; } \\
1, & \text { otherwise }\end{cases}
\end{gathered}
$$




\section{Vertical Links}

$$
\begin{aligned}
& A_{v}^{Y} \text { for } 1 \leq i \leq m \text { and } 1 \leq j \leq(n+1) \text { represents matrix of vertical } \\
& \text { links of the network }
\end{aligned}
$$

$$
A_{j}^{y}= \begin{cases}1, & \text { if the vertical link connecting nodes }(i, j) \text { and }(i+1, j) \\ 0, & \text { otherwise }\end{cases}
$$

(b)

$$
\begin{aligned}
& Y_{i j} \text { for } 1 \leq i \leq m \text { and } 1 \leq j \leq(n+1) \text { represents matrix of lengths of } \\
& \text { vertical }
\end{aligned}
$$

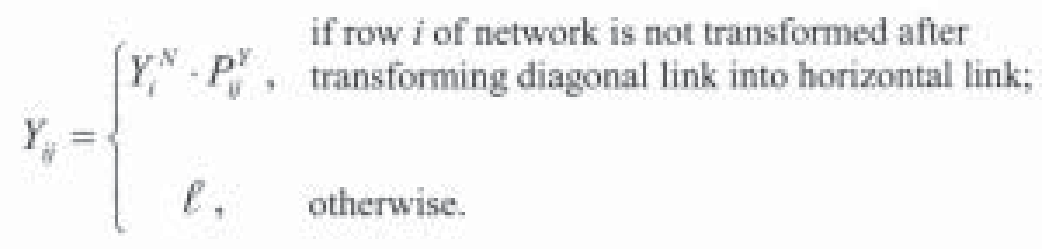

where:

$P_{y}^{\gamma} \quad$ takes the value of 1 or $+\infty$ depending on whether the vertical link $(i, j)$ is a real or dummy link.

$Y_{i}^{N} \quad$ represents height of the row $i$.

$\ell \quad$ is the length of a vertical link that was introduced after transformation of the diagonal link

As discussed in the previous section, each diagonal link is transformed into a horizontal link, and the row where the diagonal link was is split into two geometrically equal rows. There are three types of vertical links in the two new rows that replaced the original row:

i. A vertical link that represents a point where a diagonal link is beginning or ending (e.g., links $A X$ and YD in transformed network shown in Figure $1 \mathrm{~b}$ represent points $A$ and $D$ in original network shown in Figure 1a). Value of $\ell$ for these links is equal to 0 . 
ii. A new vertical link that represents the original vertical link leading to/from diagonal link (e.g., links $X C$ and $B Y$ in Figure $1 b$ represent links $A C$ and $B D$ in the original network in Figure 1a). Value of $\ell$ for these links will be equal to original height of the initial row containing the diagonal link.

iii. A vertical link that is not connected with the diagonal link; but is in the transformed row. Value of $\ell$ for such vertical links is equal to half of the original row height if the vertical link was a real link, or equal to $+\infty$ if the vertical link was a dummy link (e.g., link PQ in the Figure $1 \mathrm{~b}$ is equal to one half of the link PR in Figure 1a).

(c) $\quad P_{i j}^{\gamma}$ for $1 \leq i \leq m$ and $1 \leq j \leq(n+1)$ represents penalty matrix for vertical links

$$
P_{i y}^{Y}= \begin{cases}+\infty, & \text { if the vertical link connecting nodes }(i, j) \text { and }(i+1, j) \\ 1, & \text { otherwise }\end{cases}
$$

\section{Nodes}

$$
B_{i j} \text { for } 1 \leq i \leq(m+1) \text { and } 1 \leq j \leq(n+1) \text { represents matrix of network }
$$
nodes

$$
B_{j}= \begin{cases}1, & \text { if the node }(i, f) \text { is in the bus route; } \\ 0, & \text { otherwise. }\end{cases}
$$

(b) $\quad T_{i j}$ for $1 \leq i \leq(m+1)$ and $1 \leq j \leq(n+1)$ represents matrix of intersection delay times for node $(i, j)$ 
Since the passenger demand of zone $(i, j)$, denoted by $q_{i j}$, to the CBD is known, the total passenger demand $(Q)$ can be calculated as:

$$
Q=\sum_{i=1}^{m} \sum_{j=1}^{n} q_{j, j} \quad \text { for } m \geq 1 \text { and } n \geq 1
$$

The objective function of the analyzed bus routing problem is the total system cost $\left(C_{T}\right)$, including operator cost $\left(C_{s}\right)$ and user cost $\left(C_{u}\right)$. Thus,

$$
C_{r}=C_{s}+C_{u}
$$

The operator cost, in dollars per hour, is equal to the fleet size $(F)$ multiplied by the bus operating cost $\left(u_{B}\right)$ :

$$
C_{s}=F \cdot u_{B},
$$

The bus operating cost can be estimated from the average wage rate labor, insurance, and maintenance expenses. Required fleet size can be estimated from the vehicle round-trip time $\left(T_{R}\right)$ divided by the headway $\left(H_{B}\right)$. Thus,

$$
F=\frac{T_{R}}{H_{B}}
$$

In Equation (4) the round trip travel time $\left(T_{R}\right)$ is double the sum of bus route travel time $\left(T_{L}\right)$, total route intersection delay $\left(T_{D}\right)$, and line-haul travel time $\left(T_{J}\right)$, while the vehicle layover time is negligible here. Therefore,

$$
T_{R}=2\left(T_{L+} T_{D+} T_{J}\right)
$$


The total local route travel time $\left(T_{L}\right)$ is defined as:

$$
T_{L}=\frac{1}{V_{B}}\left[\sum_{j=1}^{m} \sum_{j=1}^{n+1} A_{j}^{\gamma} Y_{j}+\sum_{j=1}^{m+1} \sum_{j=1}^{n} A_{i j}^{x} X_{i j}\right] \text {, for } m \geq 1 \text { and } n \geq 1
$$

where:

$V_{B} \quad$ is the average bus operating speed on local streets.

The average intersection delay time Tij incurred by buses can be estimated from the field data. Thus, the total intersection delay $\left(T_{D}\right)$ per bus trip can be formulated as:

$$
T_{D}=\sum_{j=1}^{n+1} \sum_{j=1}^{n+1} B_{i j} \cdot T_{i j}
$$

The bus line-haul travel time, denoted by $T_{J}$, is equal to the line-haul distance $\left(L_{J}\right)$ divided by the line-haul speed $\left(V_{J}\right)$ :

$$
T_{J}=\frac{L_{J}}{V_{J}}
$$

The user cost considered in this study consists of three elements: user access cost $\left(C_{A}\right)$, user wait cost $\left(C_{W}\right)$, and user in-vehicle cost $\left(C_{V}\right)$ :

$$
C_{U=} C_{A+} C_{W+} C_{V}
$$


User access cost $C_{A}$, incurred by passengers walking to the bus route, is defined as the product of user access time for each zone $(i, j)$, denoted by $a_{i j}$, and user access cost $u_{A}$ (i.e., the value of access time):

$$
C_{A}=u_{A} \sum_{j=1}^{m} \sum_{j=1}^{n} a_{j}, \quad \text { for } m \geq 1 \text { and } n \geq 1
$$

It is assumed that the passengers from zone-always walk the shortest distance- to access the bus route. The minimum distance between zone-and the access point can be estimated from the sum of horizontal and vertical distances between the gravity point of zone-and the access point. Thus, the total access time for the passengers from the zone-is:

$$
a_{i j}=\frac{D_{j} q_{i j}}{g},
$$

where:

$$
g \quad \text { denotes average passenger walking speed. }
$$

To estimate the user in-vehicle time, denoted by $C_{V}$, it is necessary to determine where passengers access the bus and how much time they spend on the bus. The first step is to identify the access point for passengers from each zone. This can be done using the shortest distance rule for calculating passenger access time. Passenger in-vehicle time is defined as the average time each passenger spends in the bus. This time is equal to total travel time including link travel times and intersection delays along the bus route. Therefore, in-vehicle time for each passenger is equal to travel time between the access point and the destination. All passengers from a particular zone will incur the same average in-vehicle time since they have the same access and destination points. Thus, total in-vehicle time for passengers from one zone is equal to the product of passenger demand for that zone and average in-vehicle time for passengers from that zone. Total passenger in-vehicle time, denoted by $S_{V}$, is simply the sum of in-vehicle times for all the zones in the 
network. And the user in-vehicle cost is equal to the product of total in-vehicle time $S_{V}$ and value of user in-vehicle time $u_{I}$ :

$$
C_{V=} S_{V} u_{I}
$$

User wait cost, the last component of total user cost, is subject to the assumption that average wait time is half of the headway. The wait cost can be formulated as:

$$
C_{W}=\frac{1}{2} H_{s} u_{W} \sum_{i=1}^{n} \sum_{j=1}^{n} q_{y} \quad \text { for } m \geq 1 \text { and } n \geq 1
$$

where:

$$
\begin{array}{ll}
\frac{1}{2} H_{B} & \text { is the average user wait time (half of headway). } \\
\sum_{i=1}^{m} \sum_{j=1}^{n} q_{i j} & \text { equals total passenger boarding. } \\
u_{W} & \text { the value of passenger wait time. }
\end{array}
$$

\section{Total System Cost - $C_{T}$}

The objective of this study is to minimize the total system cost. The objective total cost function is:

$$
C_{T=} C_{S+} C_{A+} C_{V+} C_{W}
$$

where:

$C_{S}, C_{A}, C_{V}$, and $C_{W}$ can be obtained from Equations (3), (10), (12) and (13), respectively. 
Since the decision variables in $C_{T}$ include $A_{J}^{Y}, A_{j j}^{X}, B_{I J}$, and, $H_{B}$ the total cost function can be expressed as:

$$
\begin{aligned}
& C_{T}\left(A_{j}^{y}, A_{j}^{x}, B_{U}, H_{g}\right) \\
& \text { for } 1 \leq i \leq m, 1 \leq j \leq n, 1 \leq I \leq(m+1), 1 \leq J \leq(n+1) .
\end{aligned}
$$

The optimal bus headway can be optimized if $A_{y}^{Y}, A_{f y}^{X}$, and $B_{I J}$ (elements of bus route location) are treated as exogenous variables. The optimal headway is found by setting the first derivative of the total cost function with respect to headway $\left(H_{B}\right)$ equal to zero, as formulated in Equation (16), and solving it.

$$
\frac{\partial C_{T}}{\partial H_{B}}=0
$$

Thus, the optimal bus headway $H_{B}$ is derived as:

$$
H_{B}=\sqrt{\frac{2 T_{R} u_{i j}}{\sum_{j=1}^{n} \sum_{j=1}^{n} q_{j i} u_{w}}} \text { for } m \geq 1 \text { and } n \geq 1
$$

Since all variables are nonnegative, the second derivative of the total cost function with respect to $H_{B}$ is always positive. The objective function $C_{T}\left(A_{j,}^{Y}, A_{j}^{x}, B_{u}, H_{g}\right)$ is thus convex, and a unique optimal headway exists for any given matrices $A_{J}^{Y}, A_{j j}^{X}$, and $B_{I J}$. Therefore, the minimum total system cost can be obtained by substituting the optimal headway into Equation 15.

The optimal headway must meet the route capacity constraint that states that the total route capacity should satisfy (i.e., be at least equal to) the peak-passenger demand. Thus,

$$
\frac{1}{H_{B}} \cdot C \geq Q \text {, }
$$


where:

$$
\text { C represents vehicle capacity. }
$$

The value of $H_{B}$ must satisfy the following condition:

$$
H_{s} \leq \frac{C}{Q}
$$

Therefore, the headway will decrease if the demand $Q$ increases. Since the reduced headway may increase the fleet size, as well as the operator cost, another approach to total cost optimization would be to change the bus size. This, in turn, may cause the change in the $u_{B}$, while the headway $H_{B}$ and the bus route configuration need to be reoptimized.

\section{Solution Method}

The first step in finding the optimal solution is to identify all candidate bus routes. The route location depends on the shape and size of the street network, but even for relatively small networks it is necessary to develop an algorithm and computer program to compute the optimal solution. The Exhaustive Search (ES) algorithm [11] is applied to determine the optimal bus route location. The algorithm defines all possible bus routes in the network by altering matrices $A_{v}^{t}, A_{5}^{x}$, and $B_{I J}$; optimizes headway; calculates the total cost of each candidate route; and then finally selects the optimal solution with the minimum total cost. Routes containing at least one dummy link are not considered candidate routes. A computer program is developed to process the network geometry and demand data, apply the ES algorithm, and generate the optimal solution. The program used network geometry data that can be extracted from the existing Geographic Information System (GIS) database. The program can be modified to directly retrieve input data about streets (links), intersections (nodes), and zones (street blocks) from the GIS database. This would automate the computational process, and at the same time, existing geographic data would be used as an input of the model. Another improvement of the code could, after the optimization is completed, enable the optimal solution to be uploaded into the GIS database, and then the optimal bus route can be displayed graphically. 


\section{An Example}

To illustrate the application of the model developed in this study, a numerical example is designed based on the street network with given geometric and demographic data over the service region. The analyzed network is similar to many urban commuter networks (e.g., Washington, D.C.; Chicago; Houston; and Manhattan Island in New York City, etc., as shown in Figure 2) where this model can be applied if real-world data are available. These networks usually consist of rectangular zones with diagonal thoroughfares. As mentioned before, the developed model can become even more efficient if it is integrated with the GIS database.

Figure 2. Examples of the Grid Street Networks with Diagonal Links: (a) Chicago, IL; (b) Washington, DC; (c) Manhattan Island, New York City

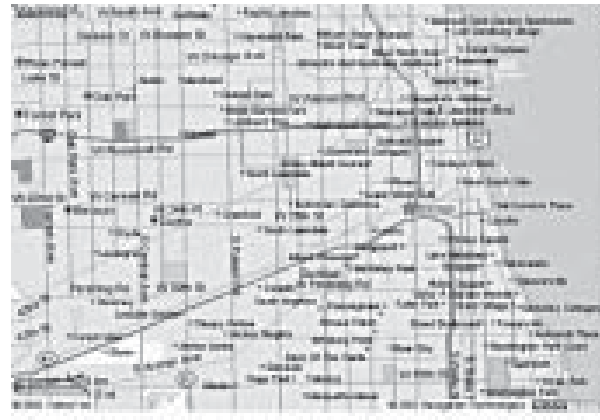

a)

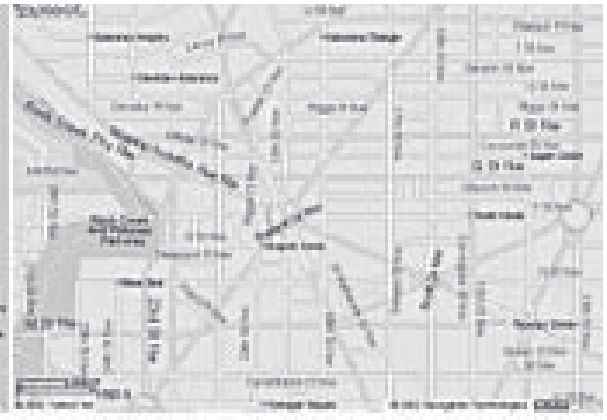

b)

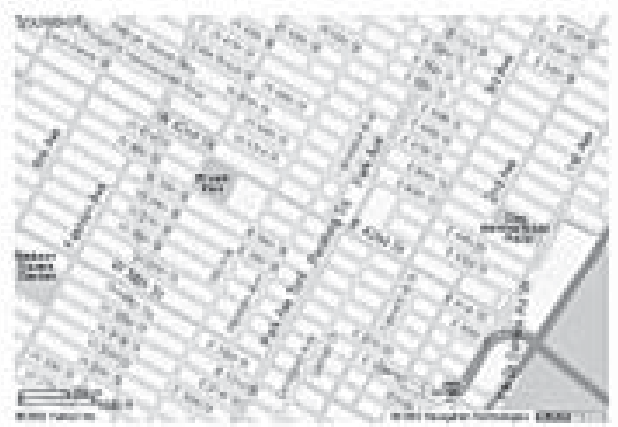

c) 
To demonstrate the performance of the developed model, a hypothetical example is designed. The analyzed service area with an underlying street network is shown in Figure 3a. The width of the service area is $1.57 \mathrm{~km}$, while the length is 2.24 $\mathrm{km}$. The network consists of $m$ (8) rows and $n$ (7) columns making 56 zones. Passenger demand for each zone is given and varies from one zone to another. Total demand for the service region is 133 passengers per hour. Buses enter the network from the left (west) and move eastward on the way to the CBD. The buses operate as a local service over the service area, and express service (no stops) is assumed from the end of the service area to the CBD. Total length of the express leg is $6.43 \mathrm{~km}$.

Figure 3a: Case Study Network (in millions)

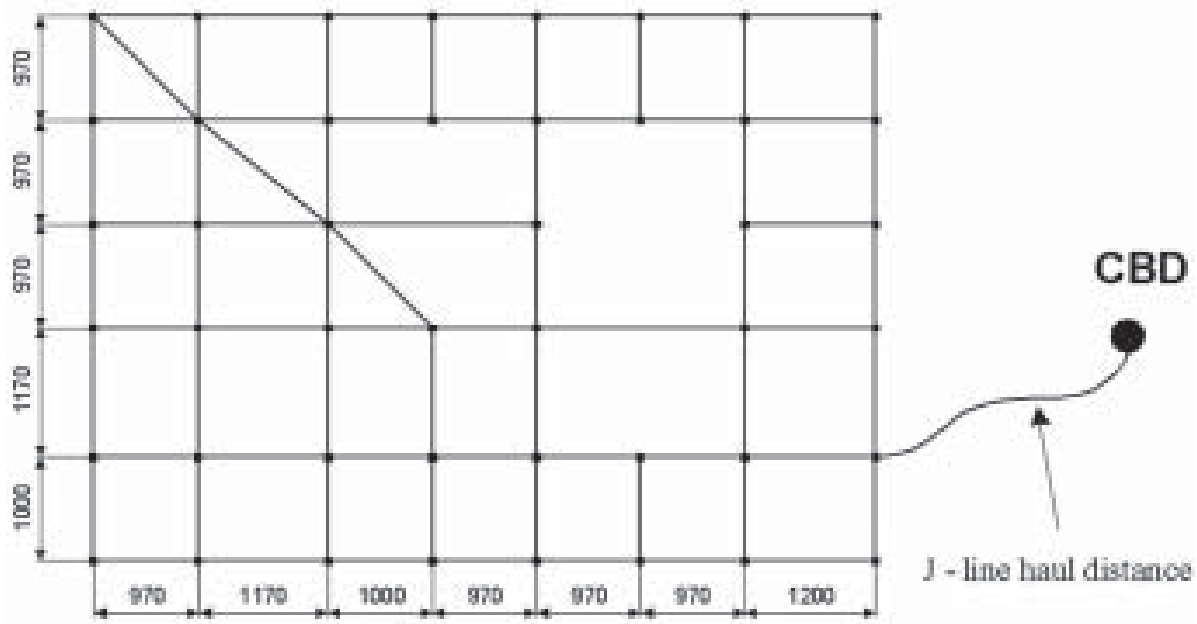

There are three diagonal links in the network. These links are transformed into horizontal and vertical links to preserve the pure grid structure (Figure 3b; the gray-colored lines showing diagonal links are not part of the modified network). The lengths of the diagonal links going from the northwest to the southeast corner are $0.43 \mathrm{~km}, 0.47 \mathrm{~km}$, and $0.43 \mathrm{~km}$, respectively. The delays at intersections are given and vary between 30 and 45 seconds per vehicle. All the other parameters of the network are given in Table 2. 
Figure 3b: Modified Case Study Network (in millions)

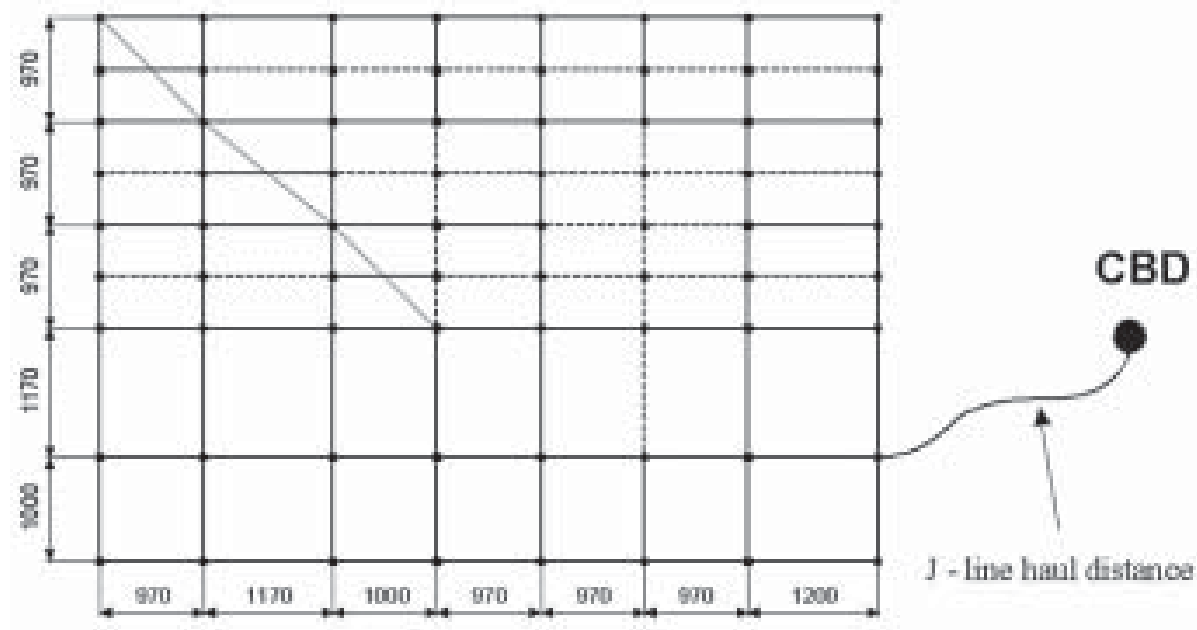

Table 2. Values of Parameters in the Case Study Network

\begin{tabular}{|lll|}
\hline Variable & Description & Value \\
\hline$u_{B}$ & A verage bus operating cost & $\$ 70.00 /$ veh-hour \\
$u_{A}$ & Value of passenger access time & $\$ 10.00 /$ pass-hour \\
$u_{W}$ & Value of passenger wait time & $\$ 10.00 /$ pass-hour \\
$u_{I}$ & Value of passenger in-vehicle time & $\$ 5.00 /$ pass-hour \\
$C_{v}$ & Bus capacity (bus size) & 50 pass $/$ veh \\
$L_{J}$ & Line-haul distance & $6.43 \mathrm{~km}$ \\
$V_{J}$ & Bus line-haul speed & $50 \mathrm{~km} / \mathrm{hour}$ \\
$V_{B}$ & Bus speed on network streets & $40 \mathrm{~km} / \mathrm{hour}$ \\
$g$ & Passenger access speed to bus route & $3.2 \mathrm{~km} / \mathrm{hour}$ \\
\hline
\end{tabular}

After the ES algorithm is performed, the objective total cost function is minimized. The optimal bus route is denoted as Route $A$ and shown in Figure 4a. The total route length is found to be $2.5 \mathrm{~km}$ long with the optimal headway of $14.2 \mathrm{~min}$ utes. The one-way travel time per trip including the express line-haul portion is 15.5 minutes and the minimum total cost is $\$ 486 /$ hour. 
Figure 4a: Optimal Bus Route A

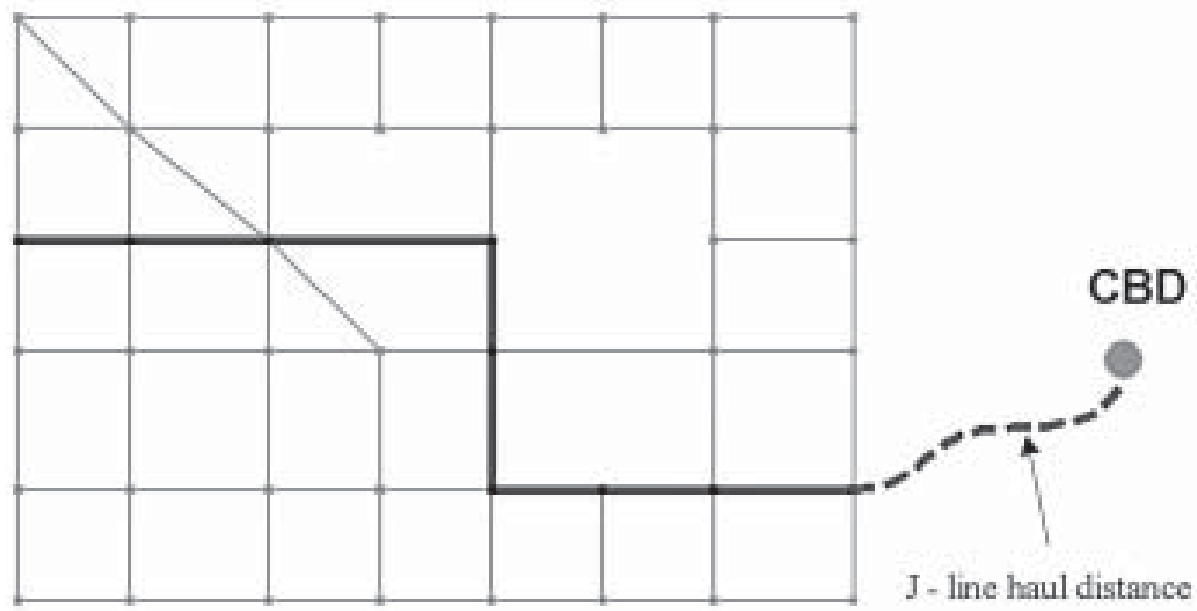

The fleet size necessary to operate on the optimal route with the optimal headway is 2.2 buses. Since in reality the number of buses must be an integer, the fleet size is arbitrarily rounded to two and three buses. The final headway is recalculated based on the new fleet sizes ( 2 or 3 buses). The fleet with three buses can be operated at the headway of 10.6 minutes achieving the total cost of $\$ 499 /$ hour. On the other hand, the fleet with two buses can be operated at the headway of 15.9 minutes and requires $\$ 488 /$ hour. The optimal fleet size of two buses has been chosen.

\section{Sensitivity Analysis}

A sensitivity analysis is performed to investigate how the model reacts to variations in the values of different parameters. Three parameters are analyzed:

1. Bus size: Three bus sizes are considered: 35,50 , and 70 passengers per vehicle, respectively. The average hourly bus operating costs vary with bus size.

2. Demand: For each zone in the network, the demand ranges from 70 percent to 150 percent of its original value.

3. Value of passenger time: Passenger wait time and access time are assumed identical and their values vary from $\$ 10$ to $\$ 15 /$ passenger-hour, while the passenger in-vehicle time ranges from $\$ 4$ to $\$ 10 /$ passenger-hour. For this calculation, the value of in-vehicle time is $\$ 5$ less than the value of wait time. 
The bus route location does not change with a variation in the value of passenger time. The same holds when the demand is increased by 50 percent. However, if the demand is increased by more than 50 percent, the configuration of the bus route changes. The new bus route is shown in Figure $4 \mathrm{~b}$ and labeled as Route $B$. Route $B$ is $2.93 \mathrm{~km}$ long, operates at the optimal headway of 10.2 minutes and has a one-way trip time, including the line haul, of 17.4 minutes. Total cost is $\$ 837 /$ hour. This change of route configuration indicates that the model is sensitive to variations in demand. Route $B$ is longer than Route $A$, and this increase resulted in an increase in in-vehicle cost. However, the reduction in access cost caused by the route relocation offsets this increase in in-vehicle cost.

Figure 4b: Optimal Bus Route B

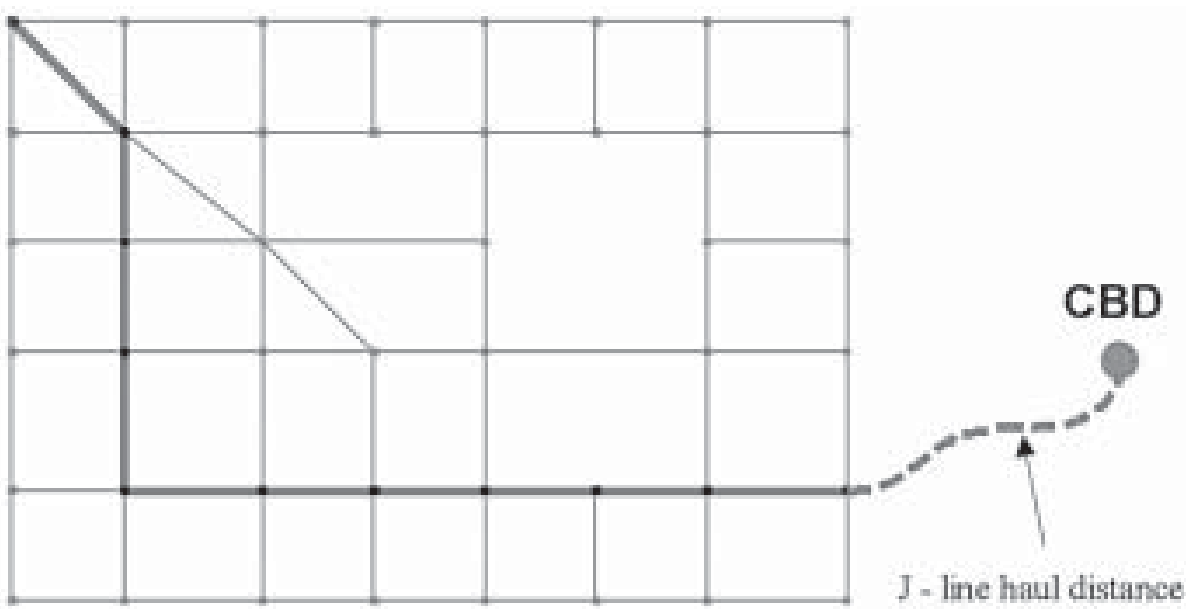

The impact of the change in headway on user, operator, and total costs is also analyzed (Figure 5). Short headway resulting in high operator costs (due to large fleet size required) reduces user costs because of less waiting time. The optimal headway is reached in point B (14.2-minute headway), at which the minimum total cost is achieved, while the operator and user costs are $\$ 159 /$ hour and $\$ 327 /$ hour, respectively.

Figure 6 shows the relationship between demand and optimal headway. For various bus sizes, the optimal headway decreases as the demand increases. Analysis results show that regardless of the variation in demand or in the value of passenger 


\section{Insert Figure 5: System Cost Versus Headway}

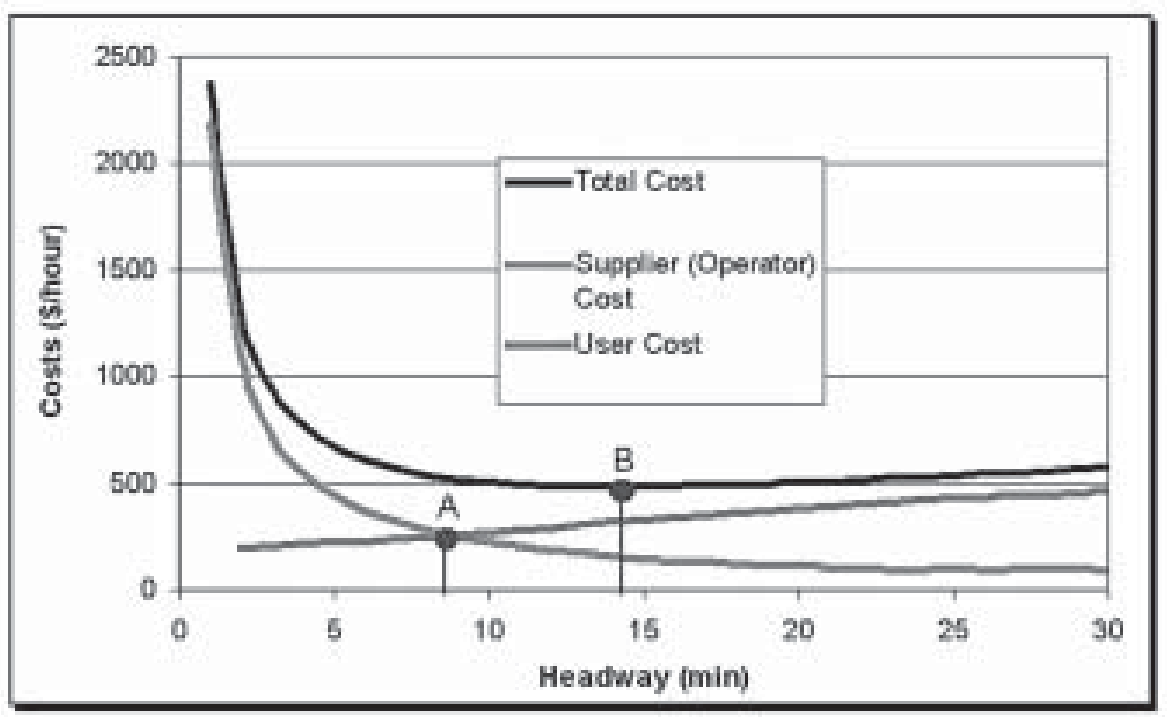

time, the 35-passenger-per-bus vehicle size is the most preferable as it yields the minimum total cost. Figure 7 shows that even change of headway does not change the optimality of using smaller buses to serve the analyzed region. In addition, Figure 8 shows that the increase in value of passenger time results in an increase in user cost. Thus, the optimal headway decreases. 
Figure 6: Optimal Headway Versus Demand

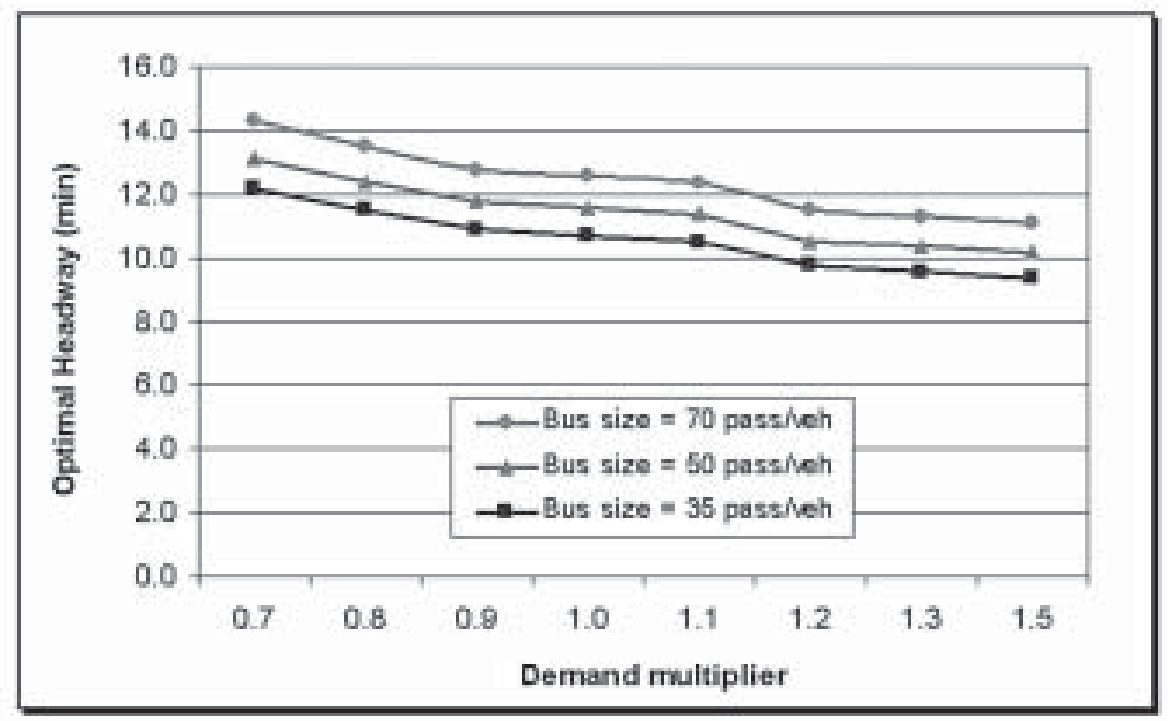

Figure 7: Total Cost Versus Headway for Various Bus Sizes

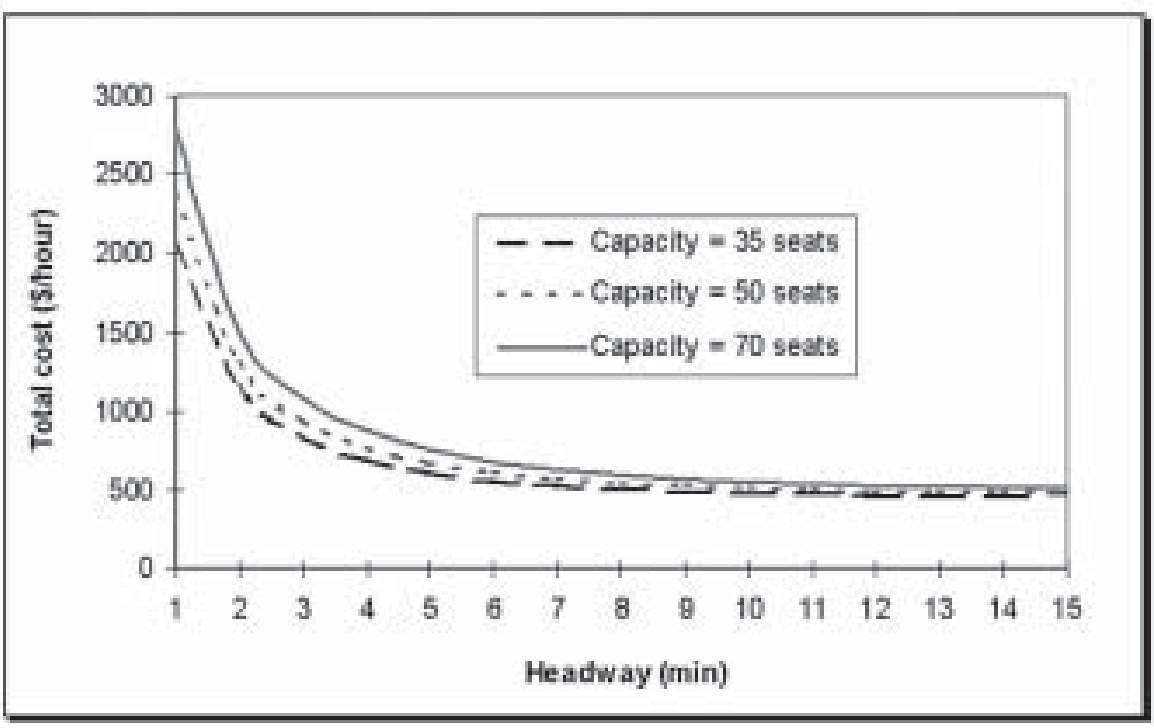




\section{Figure 8: Total Cost Versus Headway for Different Passenger Values of Time}

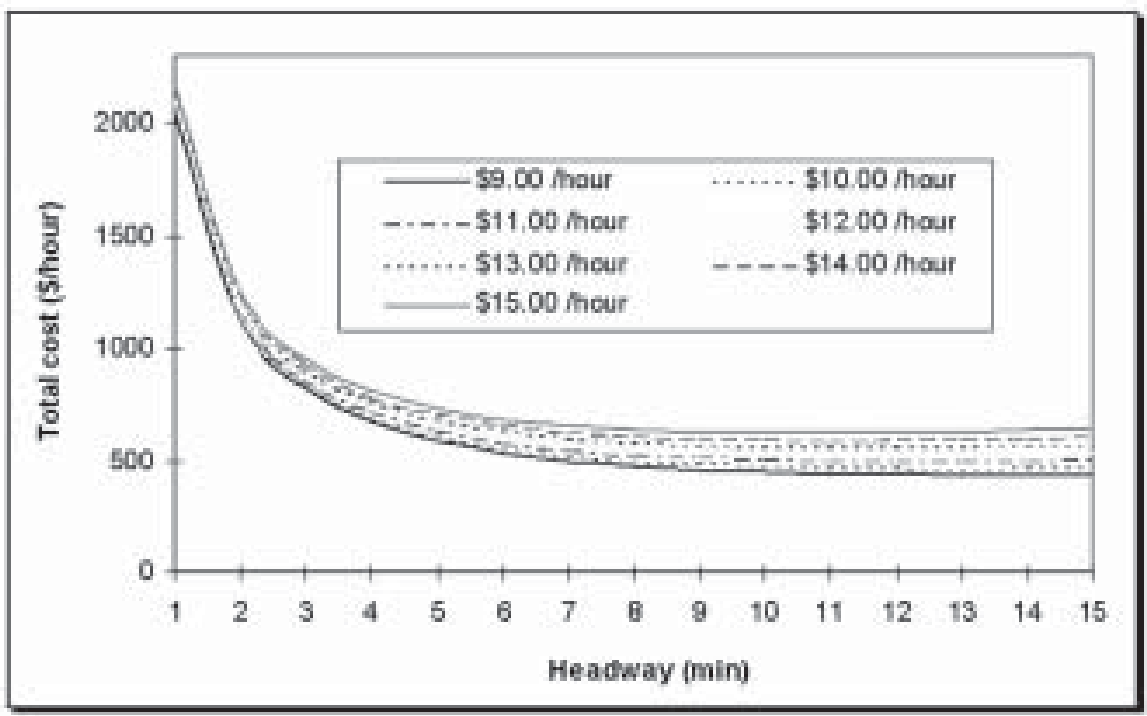

\section{Conclusions}

This article has presented a model for optimizing bus route in an urban commuter network, while considering a more realistic street pattern and demand distribution. The network transformation procedure developed in this study facilitates the models dealing with grid networks to be applied to irregular grid networks.

The model enables transit operators to optimize bus route and headway while enhancing efficient fleet management. Results derived from the model are easy to interpret and support effective decision making. Through sensitivity analysis different demand and supply conditions were evaluated by varying the optimal bus route so that it is sensitive to demand distributions, as well as value of time. Thus, the model and developed computer program are able to efficiently search for optimal solution in various conditions. The model can be easily modified to account for changes of spatial (e.g., one-way street, roadway/lane closure, reversible lane) and temporal (e.g., incidents, special events) conditions. All these features enhance transit planners' capability to redesign bus routes in areas that may experience significant shifts in residential density, as well as geographic or physical changes of the street network. 
The total cost minimization model proposed in this article may be used iteratively with a demand reestimation model to ensure that the bus system is optimized for equilibrium demand. A model that analytically integrates supply system optimization with the demand equilibration approach (e.g., as in Chang and Schonfeld [1993] or Kocur and Hendrickson [1992]) would be a desirable extension to the proposed model. It can also be further improved by introducing multiple bus routes to optimization procedure, which would make the model even more realistic and applicable.

A very important extension of this research is design of an interface with GIS databases containing real-world information on street geometry, demography, and traffic conditions in the studied service area. This information includes average vehicle operating speeds in different zones, street patterns, rights-of-way, street directionality, and time and spatial distribution of passenger demand. The GIS interface would enable data exchange between model and GIS: input data would be automatically retrieved from the GIS database, and the solution would be geocoded, loaded back into database, and displayed using graphic capabilities of the GIS software. This would improve analysis and let the user easily browse through different scenarios and corresponding solutions and to compare them.

\section{Acknowledgments}

This research was partially supported by a grant from the U.S. Department of Transportation, University Transportation Centers Program, through the National Center for Transportation and Industrial Productivity (NCTIP) at New Jersey Institute of Technology (NJIT). This support is gratefully acknowledged but implies no endorsement of the conclusions by these organizations. 


\section{References}

Byrne, B. F., and V. Vuchic. 1971. Public transportation line positions and headways for minimum cost. In Traffic Flow and Transportation, 347-360. New York: Elsevier Publishing.

Chang, S. K., and P. M. Schonfeld. 1991. Optimization models for comparing conventional and subscription bus feeder services. Transportation Science 25:281298.

Chang, S. K., and P. M. Schonfeld. 1993. Welfare maximization with financial constraints for bus transit systems. Transportation Research Record 1395:48-57.

Chien, S., and P. M. Schonfeld. 1997. Optimization of grid transit system in heterogeneous urban environment. Journal of Transportation Engineering 123(1):2835.

Chien, S., and P. M. Schonfeld. 1998. Joint optimization of a rail transit line and its feeder bus system. Journal of Advanced Transportation 32(3):253-284.

Chien, S., and Z. Yang. 2000. Optimal feeder bus routes with irregular street networks. Journal of Advanced Transportation 34(2):213-248.

Hurdle, V. F. 1973. Minimum cost locations for parallel public transit lines. Transportation Science 7:340-350.

Kocur, G., and C. Hendrickson. 1992. Design of local bus service with demand equilibrium. Transportation Science 16(2):149-170.

Spasovic, L. N., and P. M. Schonfeld. 1993. A method for optimizing transit service coverage. Transportation Research Record 1402:28-39.

Spasovic, L. N., M. P. Boile, and A. K. Bladikas. 1994. Bus transit service coverage for maximum profit and social welfare. Transportation Research Record 1451:1222.

Welch, W., R. Chisholm, D. Schumacher, and S. R. Mundle. 1991. Methodology for evaluation out-of-direction bus route segments. Transportation Research Record 1308:43-50.

Wirasinghe, S. C., V. F. Hurdle, and G. F. Newell. 1977. Optimal parameters for a coordinated rail and bus transit system. Transportation Science 11:359-374. 


\section{About the Authors}

Steven I-Jy Chien (chien@adm.njit.edu) is an associate professor of civil engineering and has a joint appointment with the interdisciplinary program in transportation at NJIT. Dr. Chien earned his master's and Ph.D. degrees from the University of Maryland at College Park.

His teaching interests are in public transportation systems, transportation planning, traffic simulation, and urban systems engineering. His research interests are focused on intermodal transit systems, microscopic traffic simulation, transportation systems analysis, intelligent transportation systems, and application of artificial intelligence in transportation problems.

Branislav V. DimitriJeVic (bxd1947@njit.edu) is a research assistant and Ph.D. candidate in the interdisciplinary program in transportation at NJIT. He earned his master's degree from NJIT and his bachelor's degree from the University of Belgrade, Yugoslavia. His current research areas are network optimization, multimodal freight transportation, and transportation systems analysis.

LazAR N. Spasovic (spasovic@njit.edu) is a professor of transportation and management at NJIT and director of the National Center for Transportation and Industrial Productivity (NCTIP) and the International Intermodal Transportation Center (IITC). He is codirector of the Brownfields Economic Redevelopment initiative.

A recognized expert in intermodal freight transportation and transportation system analysis, Dr. Spasovic holds a Ph.D. degree in systems engineering from the University of Pennsylvania. He teaches multimodal freight transportation and urban transportation planning. 